\title{
Le Tournant réflexif, naissance d'In Situ. Au regard des sciences sociales
}

The reflexive turn, birth of In Situ. Au regard des sciences sociales

Isabelle Chave et Christian Hottin

\section{(2) OpenEdition}

\section{Journals}

Édition électronique

URL : https://journals.openedition.org/insituarss/286

DOI : $10.4000 /$ insituarss. 286

ISSN : 2680-4972

Éditeur

Ministère de la Culture

\section{Référence électronique}

Isabelle Chave et Christian Hottin, «Le Tournant réflexif, naissance d'In Situ. Au regard des sciences sociales », In Situ. Au regard des sciences sociales [En ligne], 1 | 2019, mis en ligne le 15 octobre 2019 consulté le 16 décembre 2022. URL : http://journals.openedition.org/insituarss/286 ; DOI : https:// doi.org/10.4000/insituarss.286

Ce document a été généré automatiquement le 16 décembre 2022.

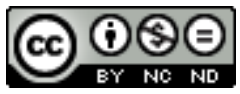

Creative Commons - Attribution - Pas d'Utilisation Commerciale - Pas de Modification 4.0 International - CC BY-NC-ND 4.0

https://creativecommons.org/licenses/by-nc-nd/4.0/ 


\title{
Le Tournant réflexif, naissance d'In Situ. Au regard des sciences sociales
}

\author{
The reflexive turn, birth of In Situ. Au regard des sciences sociales
}

\author{
Isabelle Chave et Christian Hottin
}

1 Pour le ministère de la Culture, le patrimoine est une cause que l'on sert. Telle est la mission de l'institution, et c'est ainsi qu'elle est vécue, chaque jour, grâce à la passion qui anime ses membres. Reste que cette cause ne mériterait sans doute pas plus d'une minute d'attention, si le patrimoine n'était aussi, à chaque instant, une question que l'on se pose. Une idée mise en discussion, remise en question, une notion dont on explore les contours et dont on cherche à saisir les métamorphoses. Telle est l'ambition de cette nouvelle publication : In Situ. Au regard des sciences sociales.

2 Née avec le siècle quant à elle, In Situ. Revue des patrimoines entre dans sa dix-huitième année ${ }^{1}$. Conçue à l'origine comme une publication périodique exclusivement en ligne pour la mise en valeur des travaux des chercheurs de l'Inventaire général et, à ce titre, attachée à ce service du ministère (Verdier 2001), elle a connu au fil des ans de nombreuses transformations, ne cessant d'élargir son périmètre, de diversifier ses champs disciplinaires, d'étoffer son comité de rédaction. Plusieurs de ces changements sont liés aux rapprochements vécus par les membres de différentes administrations centrales, jusqu'à la constitution d'une direction générale des Patrimoines en 2010. Archéologues, ethnologues, conservateurs de Monuments historiques ont rapidement rejoint le projet, suivis quelques années plus tard par des collègues des musées, des archives, des bibliothèques et de plusieurs établissements publics. Les chercheurs universitaires ont toujours été présents au sein du comité et des collègues des universités comme du CNRS ont, depuis toujours, pris part aux dossiers thématiques de la revue. Les démarches prospectives et réflexives, présentes dès les premières années (on songe ainsi au $\mathrm{n}^{\circ} 3$, «Les horizons de l'Inventaire » en $2003^{2}$ ), se sont affirmées plus récemment, par exemple à travers les livraisons dédiées au patrimoine culturel immatériel $\left(n^{\circ} 33,2017^{3}\right)$ ou à l'histoire et au fonctionnement des métiers du patrimoine $\left(\mathrm{n}^{\circ} 30,2016^{4}\right)$. 
3 Lorsque parurent ces deux numéros en 2016-2017, In Situ. Revue des patrimoines cheminait déjà depuis quelques années aux côtés de la recherche en ethnologie de la France et des publications que le ministère consacre à ce domaine, toutes créées en 1983 : la revue Terrain et les deux collections que sont les «Cahiers d'ethnologie de la France » et "Ethnologie de la France ». Si Terrain", emmené par sa rédactrice en chef Christine Langlois et son comité de rédaction, a souhaité affirmer toujours plus clairement son orientation généraliste, passant de l'appellation «Carnets du patrimoine ethnologique » à celle de "Revue d'ethnologie de l'Europe » et complétant son titre par «Anthropologie et sciences sociales", les collections, éditées par les Éditions de la Maison des sciences de l'homme et regroupées depuis janvier 2019 sous le titre unique "Ethnologie de la France et des mondes contemporains ${ }^{6}$ ", sont quant à elles devenues progressivement les lieux privilégiés de diffusion des recherches en ethnologie du patrimoine, un courant fortement représenté au sein du Laboratoire d'anthropologie et d'histoire de l'institution de la culture (Lahic, équipe de l'IIAC), le laboratoire co-construit par le ministère, le CNRS et l'EHESS que fonda et dirigea jusqu'à sa mort l'anthropologue Daniel Fabre. Au cours de cette même période, l'important déploiement de la Convention Unesco de 2003 pour la sauvegarde du patrimoine culturel immatériel, approuvée par la France en juillet 2006, a suscité de très nombreux travaux de recherche : en ethnologie, bien entendu, mais aussi en droit, en économie, en histoire culturelle. Cette question du «PCI » s'est bien vite imposée comme véritable laboratoire d'une approche interdisciplinaire sur les questions patrimoniales (Chave 2018).

4 En 2016, avec le départ en retraite de Christine Langlois, qui avait porté avec un talent sans égal la publication de Terrain et de la collection «Ethnologie de la France » durant plus de trente ans, s'est posée avec acuité la question de l'évolution des publications de l'ethnologie au ministère de la Culture. Il est apparu que, à côté d'un soutien diversifié aux différents acteurs de l'édition scientifique en ethnologie et anthropologie, le temps était venu, sans aucun doute, de rassembler des chercheurs de différentes disciplines autour d'un projet commun d'étude des questions patrimoniales et d'organiser, sous la forme d'une publication périodique, des rencontres et des réflexions qui se produisaient auparavant au gré des circonstances, à l'occasion de colloques et de programmes de recherche.

5 Il n'est pas surprenant que le besoin s'en fasse précisément sentir, alors que l'approche pluridisciplinaire du fait patrimonial connaît une vraie dynamique. En témoignent par exemple la création du blog Respatrimoni, qui fédère depuis juin 2009, quel que soit leur rattachement académique, le réseau des chercheurs sur les patrimonialisations ${ }^{7}$; la fondation de l'Association of Critical Heritage Studies ${ }^{8}$ (ACHS) en 2012 à Göteborg, en Suède, dont le manifeste fondateur appelait à la mise en œuvre et à la valorisation d'études critiques et d'interventions innovantes, afin d'interroger les jeux de pouvoir et les intrications économiques et culturelles sous-tendus par les définitions et les pratiques traditionnelles du patrimoine; ou encore la naissance en 2016, durant le congrès de Montréal de l'ACHS en 2016, de son Réseau francophone, qui rassemble chercheurs, enseignants et professionnels du patrimoine culturel, partageant une approche critique du patrimoine et des patrimonialisations au sein de l'association internationale?.

6 La naissance de cet In Situ. Au regard des sciences sociales s'inscrit dans un cheminement complexe, un parcours qui mêle mutations administratives et cristallisation de 
nouvelles problématiques scientifiques, à la rencontre de différentes disciplines et de différentes institutions. Aussi, cette nouvelle publication entend assumer pleinement son double héritage : celui d'In Situ « canal historique ", d'une part, auprès de laquelle elle va cheminer désormais, et celui des publications de l'ethnologie au ministère d'autre part, avec lesquelles elle partage un souci de réflexivité et d'approche critique des questions patrimoniales.

7 Dès la fin de l'année 2015, un groupe de travail s'est mis en place pour réfléchir à la ligne éditoriale de la future publication. Plusieurs pistes furent envisagées, jusqu'à retenir celle d'une revue fondée sur un socle ethnologique, mais ouverte à un large panel disciplinaire, et centrée sur l'étude des questions patrimoniales, comme un phénomène social, culturel, économique et politique et un domaine d'application d'une multiplicité de points de vue - académiques, professionnels, gouvernementaux, associatifs... Pensé dans une perspective de durabilité et d'équité sociale et culturelle ou non, le fait patrimonial se prête à une réflexion transdisciplinaire et transversale sur ses fondements discursifs et politiques. In Situ. Au regard des sciences sociales portera à la connaissance du public des recherches conduites sur l'histoire et l'évolution de l'appropriation sociale du fait patrimonial, en recherchant une représentativité des diverses disciplines, sans exclusive, représentées par les sciences humaines et sociales, et des parcours individuels: chercheurs académiques, professionnels du secteur et acteurs culturels, tous sont invités à se plonger dans l'actualité du patrimoine et à contribuer à l'analyse critique de ses constructions et de son rôle.

8 Merci à toutes celles et à tous ceux qui ont donné de leur temps, sans compter, pour prendre part à cette aventure passionnante qu'est toujours la naissance d'une nouvelle publication. Progressivement, le groupe de travail s'est mué en un comité de rédaction " en préfiguration ", puis dûment constitué. Il lui est revenu, notamment, de penser l'organisation du sommaire "type » d'un numéro, avec son dossier thématisé et ses rubriques récurrentes, d'en définir le premier thème - « Le tournant réflexif » - et d'en élaborer le programme.

Cette dynamique nouvelle, qui allait conforter la place des études sur le patrimoine au sein des sciences humaines et sociales au cours des années 1990, passe en effet pour avoir affecté, au premier chef, la discipline ethnologique ${ }^{10}$, d'autant que, comme l'a montré Jean-Louis Tornatore, qui, dès 2004, utilisait l'expression « tournant réflexif » à propos de la Mission ministérielle du patrimoine ethnologique, ce mouvement de sortie des procédures classiques de la patrimonialisation engageait, au sein de la discipline, « un double renversement de perspective : faire du patrimoine ethnologique, voire de tout patrimoine, la matière d'une ethnologie de l'activité patrimoniale; déplacer la position d'expertise au cœur de la scène patrimoniale de manière à transformer l'expertise documentaire de l'ethnologue en expertise participative » (Tornatore 2004).

Questionnant l'élargissement de ce phénomène aux sciences humaines et sociales en leur ensemble, les contributions rassemblées en ce premier dossier thématique visent donc à exposer comment l'ethnologie ou l'anthropologie, mais aussi d'autres domaines académiques donc, ont été infléchis ou touchés par la question patrimoniale et comment le fait patrimonial a influé sur le renouvellement disciplinaire. Le processus est illustré par quatre disciplines académiques: l'histoire culturelle, avec Christian Hottin ("Témoignage pour une histoire administrative de la Mission du patrimoine ethnologique »), l'anthropologie, avec Jean-Louis Tornatore («Pour une anthropologie plébéienne et pragmatiste du patrimoine: un scénario contre-hégémonique»), les 
sciences économiques, avec Francesca Cominelli et Xavier Greffe («L'économie politique du patrimoine culturel») et la géographie, avec Olivier Lazzarotti ("Cinq leçons géographiques sur les mémoires »).

11 Certes, d'autres disciplines auraient pu venir compléter ce panorama non exhaustif. Invoquons le seul exemple du droit, qui a connu une ouverture déterminante avec l'avènement du droit du patrimoine culturel, sollicitant pour une grande part les droits nationaux mais inscrit également dans un contexte international, et a renouvelé ses méthodes et ses champs de recherche, en faisant progresser la réflexion sur les concepts fondamentaux, les différents objets et modes de protection et la prise en compte des intérêts politiques ou juridiques ${ }^{11}$. Les recherches de Noé Wagener (2015) et des membres du programme de recherche Osmose en droit comparé du patrimoine culturel immatériel ${ }^{12}$, ont ainsi montré les conséquences de la saisie par le droit du patrimoine en France, au début des années 2000, de la notion de patrimoine culturel immatériel, centré sur le rôle des communautés et posant d'importants problèmes en matière de propriété intellectuelle ou industrielle.

Chaque numéro d'In Situ. Au regard des sciences sociales sera enrichi, par ailleurs, de rubriques récurrentes, dont on découvrira dans cette première livraison les quatre principales: "Études de cas", qui regroupe des varia en dehors de la logique thématique du numéro, s'ouvre à une contribution de Claudie Voisenat («Les transparences de la médiation. Anthropologie d'un patrimoine hors l'histoire»). «Situations », qui propose témoignages et récits d'acteurs, professionnels ou non, du patrimoine, est illustrée par le témoignage de Séverine Cachat, recueilli par Isabelle Chave («L'expérience du Centre français du patrimoine culturel immatériel»). "Débats», inaugurée avec un article de Noël Barbe («Pour une anthropologie plébéienne et pragmatiste du patrimoine. En finir avec le patrimoine patricien. Par le bas et par la marge »), accueille, en écho à l'actualité d'ouvrages parus, de colloques ou de séminaires, d'expositions, etc., des regards particuliers, témoignant d'un point de vue, d'une prise de position individuelle ou d'un engagement théorique, institutionnel ou pratique, sur les constructions patrimoniales. Enfin, «Comptes rendus » recense et fait le point de publications récentes, imprimées ou électroniques, et de nouvelles ressources disponibles; Léonie Hénaut («Les professionnels du patrimoine à l'épreuve du tournant patrimonial ») en est la première contributrice.

13 Ce numéro fondateur, «Le Tournant réflexif», nous le portons aujourd'hui collectivement devant les lecteurs internautes.

\section{BIBLIOGRAPHIE}

Bouju Marie-Cécile \& Hotтin Christian (dir.,), 2016 In Situ. Revue des patrimoines [en ligne], $\mathrm{n}^{\circ}$ 30, «Au regard des métiers du patrimoine », https://journals.openedition.org/insitu/13530 [lien valide en mai 2019]. 
Саснат Séverine \& HоттіN Christian (dir.), 2017 In Situ. Revue des patrimoines [en ligne], $\mathrm{n}^{\circ}$ 33, « Patrimoine culturel immatériel et institutions patrimoniales », https:// journals.openedition.org/insitu/15435 [lien valide en mai 2019].

CHAVE Isabelle, 2018 « Pratiques culturelles immatérielles. Une recherche renouvelée et décloisonnée », Culture \& Recherche, n 137, « Recherche, formation, innovation », p. 25-26. Disponible en ligne, http://www.culture.gouv.fr/Thematiques/Enseignement-superieur-etRecherche/La-revue-Culture-et-Recherche/Recherche-Formation-Innovation [lien valide en mai 2019].

CORNU Marie, FROMAGEAU Jérôme \& WALLAERT Catherine (dir.), 2012 Dictionnaire comparé du droit du patrimoine culturel, Paris, CNRS éditions, coll. «CNRS dictionnaires».

FABRE Daniel, 2016 « L'ordinaire, le familier, l'intime... loin du monument », in Christian hotTIN et Claudie VoISENAT (dir.), Le Tournant patrimonial. Mutations contemporaines des métiers du patrimoine, Paris, Éditions de la Maison des sciences de l'homme, coll. « Cahiers d'ethnologie de la France ». HоттіN Christian, 2016 « L'ethnologie, un métier du patrimoine ? Réflexions autour de la question du patrimoine culturel immatériel », In Situ. Revue des patrimoines [en ligne], $\mathrm{n}^{\circ}$ 30, « $\mathrm{Au}$ regard des métiers du patrimoine ", https://journals.openedition.org/insitu/13633 [lien valide en mai 2019].

PABoIs Marc (dir.), 2003 In Situ. Revue des patrimoines [en ligne], $\mathrm{n}^{\circ}$ 3, « Les horizons de l'Inventaire », https://journals.openedition.org/insitu/1249 [lien valide en mai 2019]. TORNATORE Jean-Louis, 2004 « La difficile politisation du patrimoine ethnologique », Terrain, $\mathrm{n}^{\circ} 42$, « Homme / femme ». Disponible en ligne, http://journals.openedition.org/terrain/1791 [lien valide en mai 2019].

VERDIER Hélène, 2001 «Éditorial. In Situ, revue de l'Inventaire général », In Situ. Revue des patrimoines [en ligne], $\mathrm{n}^{\circ}$ 1, http://journals.openedition.org/insitu/1005 [lien valide en mai 2019].

WAGENER Noé, 2015 «La Convention de 2003 à l'aune du droit français : mise en perspective historique ", communication donnée au cours de la journée d'étude « Droit comparé du patrimoine culturel immatériel » (Académie de la culture de Lettonie, Riga, 29 juin 2015). Disponible en ligne, https://www.youtube.com/watch?v=CVyoKdfjbyk [lien valide en mai 2019].

\section{NOTES}

1. Site internet de In Situ. Revue des patrimoines : https://journals.openedition.org/insitu/

2. Marc Pabois (2003).

3. Cachat \& Hottin (2017).

4. Bouju \& Hottin (2016).

5. Site de la revue Terrain, désormais accueillie par l'université Paris Nanterre: https:// journals.openedition.org/terrain/

6. Collection «Ethnologie de la France et des mondes contemporains » : http://www.fmsh.fr/fr/ diffusion-des-savoirs $/ 28588$

7. Blog Respatrimoni : https://respatrimoni.wordpress.com/

8. Site internet de l'ACHS : https://www.criticalheritagestudies.org/

9. Site internet du Réseau francophone ACHS : https://achsfr.wordpress.com/

10. «Surtout, dès le milieu des années 1990, une bonne part des ethnologues du Ministère, en administration centrale comme dans les Drac, rompt en douceur avec le postulat initial de la construction d'un patrimoine ethnologique national via le développement de la recherche en 
ethnologie de la France, pour se tourner, dans une perspective critique et réflexive, vers une " ethnologie du patrimoine », où l'institution est désormais saisie comme objet d'étude » (Hottin 2016 : §17).

11. Reflet de l'actualité des recherches sur la terminologique juridique relative au droit du patrimoine culturel en Allemagne, Angleterre, Espagne, France, Italie et Suisse, le Dictionnaire comparé du droit du patrimoine culturel (Cornu, Fromageau \& Wallaert 2012), a clarifié les différents outils normatifs au plan interne et international, dans la comparaison des systèmes et la mise en lumière des mécanismes originaux de protection du patrimoine. Sa réédition, revue et augmentée, est en cours pour 2021, élargie à six nouveaux pays (Belgique, Croatie, Grèce, Lettonie, Pays-Bas et Pologne).

12. Ces travaux sont restitués notamment sur le blog «Droit, patrimoine et culture. Nouveaux champs de recherche » de l'Institut des sciences sociales du politique (ISP-UMR CNRS 7220): https://dpc.hypotheses.org/

\section{RÉSUMÉS}

Fondateur de la revue In Situ. Au regard des sciences sociales, ce numéro 1 affirme dès son intitulé la vocation de cette nouvelle publication: proposer un regard réflexif sur le fait patrimonial, comme processus et phénomène, et faire apparaître les conséquences de sa constitution progressive en champ de recherche transversal sur le renouvellement des objets et des méthodes des sciences humaines et sociales, dans la palette disciplinaire la plus vaste possible. Les quatre articles du dossier thématique, de manière non exhaustive, en proposent des applications à l'histoire culturelle (Christian Hottin), à l'anthropologie (Jean-Louis Tornatore), aux sciences économiques (Francesca Cominelli et Xavier Greffe) et à la géographie (Olivier Lazzarotti). Quatre des rubriques récurrentes, qui caractériseront chaque numéro de cette nouvelle revue, sont inaugurées avec ce premier numéro: «Études de cas», qui regroupe des essais sous forme de varia, extérieurs à la cohérence thématique du numéro (Claudie Voisenat) ; "Situations ", qui propose témoignages et récits d'acteurs du patrimoine, sous forme d'entretiens (Séverine Cachat); "Débats", qui accueille des points de vue et des engagements, théoriques, institutionnels ou pratiques, sur les constructions patrimoniales (Noël Barbe); «Comptes rendus", qui recense et fait le point de publications récentes et de nouvelles ressources disponibles, sur tous supports (Léonie Hénaut).

Inaugural issue of the journal In Situ. Au regard des sciences sociales, this number 1 affirms the vocation of this new publication from its title: to propose a reflexive look at the patrimonial fact, as a process and phenomenon, and to reveal the consequences of its progressive constitution as a transversal field of research on the renewal of objects and methods in the human and social sciences, with the widest possible disciplinary palette. The four articles of the thematic dossier, in a non-exhaustive way, propose applications to cultural history (Christian Hottin), anthropology (Jean-Louis Tornatore), economics (Francesca Cominelli and Xavier Greffe) and geography (Olivier Lazzarotti). Four of the recurring headings, which will characterize each issue of this new journal, are inaugurated with this first issue: "Case studies", which includes essays in the form of varia, outside the thematic logic of the issue (Claudie Voisenat); "Situations", which offers testimonies and stories of heritage actors, in the form of interviews (Séverine Cachat); "Debates", which includes theoretical, institutional or practical views and commitments on 
heritage buildings (Noël Barbe); "Reports", which identifies and reviews recent publications and new resources available, in all media (Léonie Hénaut).

\section{AUTEURS}

\section{ISABELLE CHAVE}

Adjointe au chef du département du Pilotage de la recherche et de la Politique scientifique (DPRPS), direction des Patrimoines, ministère de la Culture

\section{CHRISTIAN HOTTIN}

Directeur des études de l'Institut national du patrimoine (INP), membre associé du Laboratoire d'anthropologie et d'histoire de l'institution de la culture (Lahic, IIAC, CNRS-EHESS) 